\title{
Combination treatment with light emitting diode and wound dressings in a patient with a venous leg ulcer: a case report
}

\author{
Irma Bernadette S. Sitohang ${ }^{1 凶}$, Mutiara Ramadhiani ${ }^{1}$, Karin Rachmani ${ }^{1}$, Hanif Sri Utami ${ }^{1}$, Melani Marissa ${ }^{1}$ \\ ${ }^{1}$ Department of Dermatology and Venereology, Faculty of Medicine, Universitas Indonesia, Dr. Cipto Mangunkusumo Hospital, Jakarta, Indonesia.
}

\begin{abstract}
Leg ulcers are a problem often found in the adult population, with a prevalence of 1 to $2 \%$ in the population. Vascular disease is the most common cause. Venous disorders that occur in the lower limbs are the main cause of lower leg ulcers compared to other vascular disorders. Emerging wounds can cause pain, thereby interfering with quality of life. These conditions often persist for a long time, and they can be a health problem that leads to an economic burden as a result of a long period of wound care. Several therapeutic methods that can be chosen for wound healing purposes include light-emitting diodes (LED) and wound dressings. LED can have physiological effects such as anti-inflammatory resolution, neoangiogenesis, fibroblast and epithelial proliferation, and collagen synthesis and deposition. Furthermore, wound dressings provide an optimal condition for wound healing by creating a moist and clean environment for the wound. We present the case of a 47-year-old man with a venous leg ulcer that was treated with LED and wound dressings. After the patient underwent the eighteenth LED session, combined with wound dressings, the size, depth, and pain of the venous leg ulcer decreased.
\end{abstract}

Keywords: venous leg ulcer, light-emitting diode, LED, wound dressings, wound healing, case report

Received: 22 December 2019 | Returned for modification: 28 January 2020 | Accepted: 17 February 2020

\section{Introduction}

Leg ulcers are frequently encountered problems, with a prevalence of 1 to $2 \%$ in the adult population (1). The incidence of leg ulcers is increasing with population aging and is associated with atherosclerosis occlusion risk factors such as smoking, obesity, and diabetes. In the United States, the prevalence of vascular ulcer is estimated at 500,000 to 600,000 (2). Various types of leg ulcers that are often found include static venous ulcers, diabetic ulcers, and pressure ulcers (3). Disruption of circulation in the lower limbs from superficial veins to deep veins occurs due to valve damage to the vessels, resulting in failure of the pumping of blood against gravity to the heart. This situation causes venous insufficiency, which can subsequently cause increasing vein permeabilities, leakage, and deposition of hemosiderin. This condition impairs the microcirculatory system that brings nutrients and other substances to tissues. The tissue of the lower limb thus undergoes a state of hypoxia, which then results in a venous leg ulcer (4).

Several treatments that can be given to patients with venous leg ulcers are leg elevation, compression using stockings, oral aspirin drugs, dressings, and surgery $\left(1,4^{-6}\right)$. Wound dressings have been developed to promote wound healing. There are many types of wound dressings targeting various aspects of the healing process. Wound dressings provide a moist environment and remove bacterial infection (7). The use of photodynamic therapy, including light-emitting diode (LED), has been hypothesized to play a role in the wound healing process. An LED consists of a semiconductor chip that changes electricity into a spectrum of light (8). There are several wavelengths of LED, such as red, yellow, blue, and near infrared. LED functions in wound healing through various mechanisms, such as increasing granulation tissue formation, fibroblast proliferation, collagen synthesis, neovasculature, and epithelialization (9-12).

\section{Case report}

A 47-year-old male came to the Dermatovenereology Clinic at Dr. Cipto Mangunkusumo Hospital, in Jakarta, Indonesia due to a wound on the lower left limb that had developed 1 year before admission. Initially, he complained of an erythematous lump in the lower left limb. The lump subsequently burst, resulting in a coin-sized wound. The wound became wider, and subsequently it generated an odorless clear discharge. There was intermittent pain at the lesion site. The pain was measured on the Visual Analogue Scale (VAS) and the score was 4 . The pain could occur both during activity and rest. The patient felt a cramp in his lower left limb, which particularly worsened during the night.

The back part of the lower left limb became reddish and appeared swollen. There were no complaints of fever. A history of trauma to the left leg area before the injury was denied. The patient never applied any medication to the area of the wound. A history of diabetes mellitus, hypertension, and dyslipidemia was denied.

The patient had worked as a barber for 20 years before going to the outpatient department. He cut hair while standing. The number of customers required the patient to remain standing for many hours. During the day, he stood for approximately 6 hours. He never used compression stockings.

Physical examination demonstrated a normal general state. The patient's weight was 65 kilograms, and his height was 170 centimeters. There was left groin lymph node enlargement. The local state of the distal lower left limb and dorsum pedis revealed multiple lenticular to large hyperpigmented dully erythematous plaques; the margin was circumscribed to diffuse, formed confluences and discrete, and there was venous dilatation. Non-pitting edema was observed.

The lateral part of the lower limb had an annular-shaped ulcer 
measuring $5 \times 5 \times 1 \mathrm{~cm}$. The edge of the ulcer was slightly elevated, the wall was steep, the base of the ulcer was partially composed of granulation tissue and necrotic tissue surrounded by hyperpigmented tissue, and it did not have a foul odor (Fig. 1). Clear fluid appeared to come out of the wound. The results of Gram examination on the ulcer showed that there were many leukocytes, many Gram-positive cocci, and a moderate level of Gram-negative rods. Aerobic culture and resistance were examined, and it was found
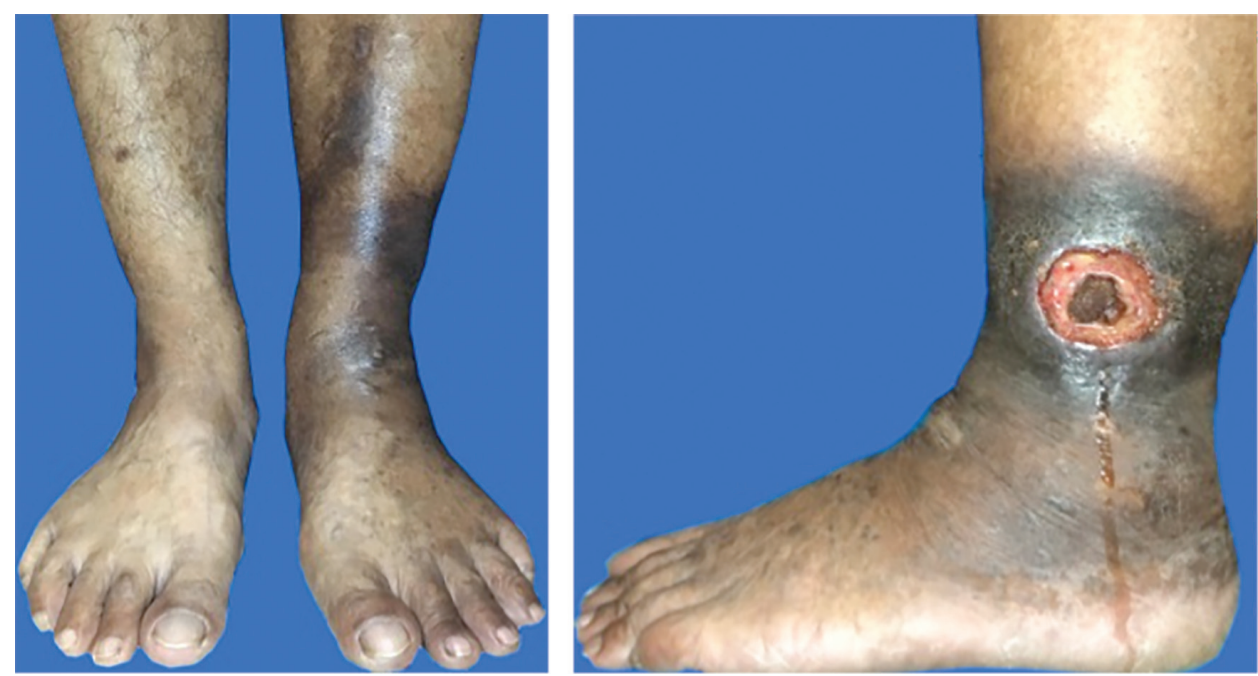

Figure 1 | Venous leg ulcer at first visit.
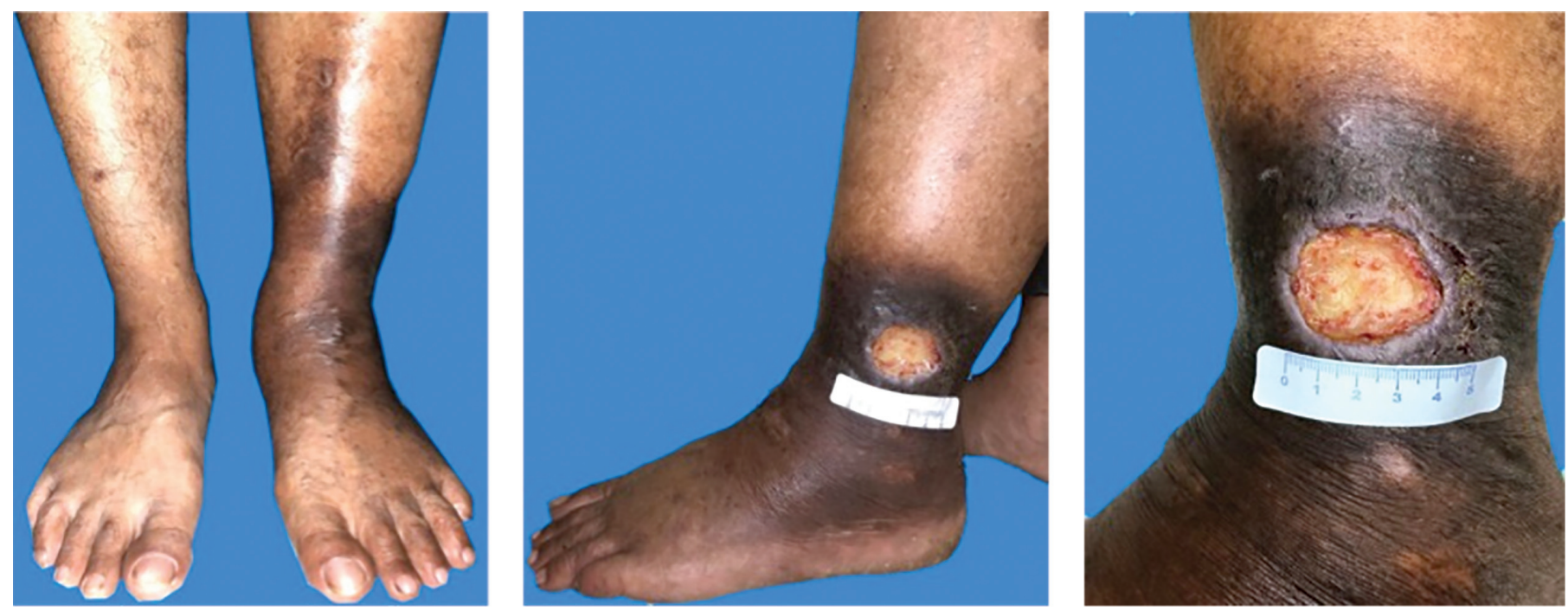

Figure 2 | Venous leg ulcer after the eighth light-emitting diode (LED) session.
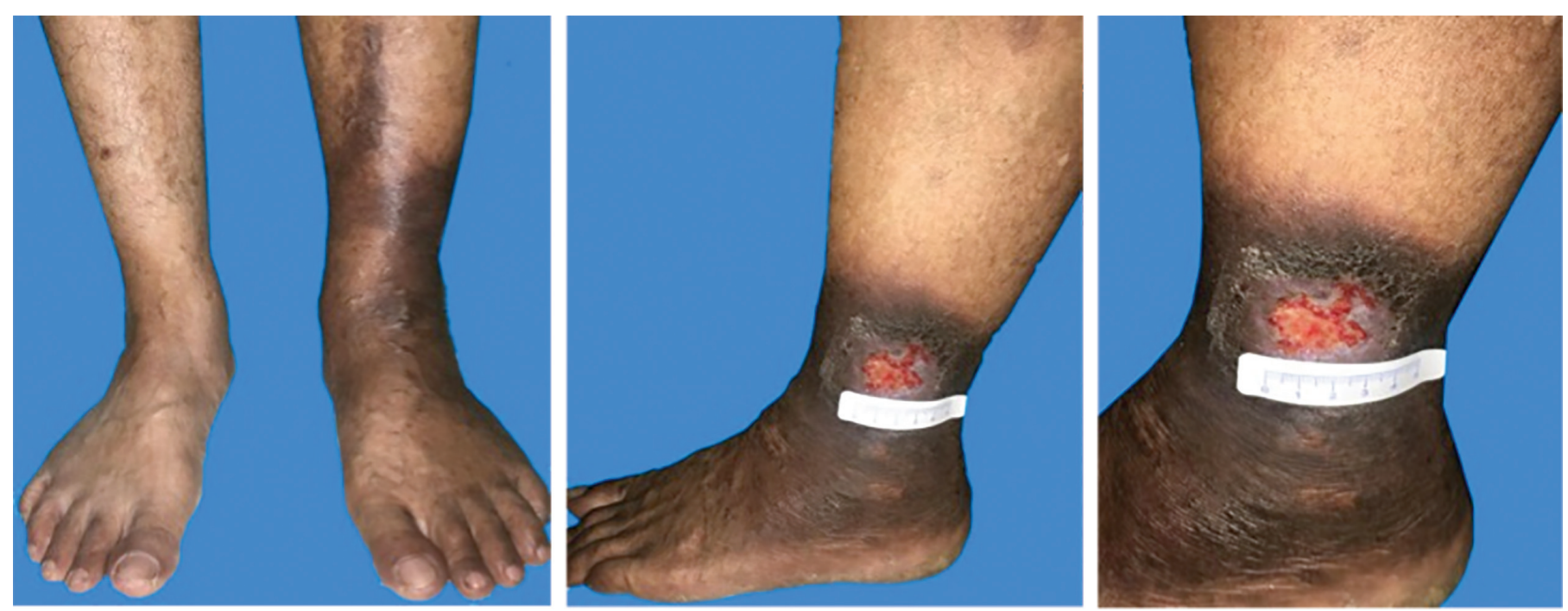

that Klebsiella oxytoca and Acinetobacter baumannii caused the infection, which were sensitive to tetracyclines and resistant to amoxicillin/clavulanic acid.

Doppler ultrasound examination was performed on the lower left leg, and it demonstrated chronic deep vein thrombosis of the rigid intraluminal material, and normal venous diameter were found. LED was performed in the wound area, with the mode of left leg. A thrombus in the common femoral and popliteal vein,

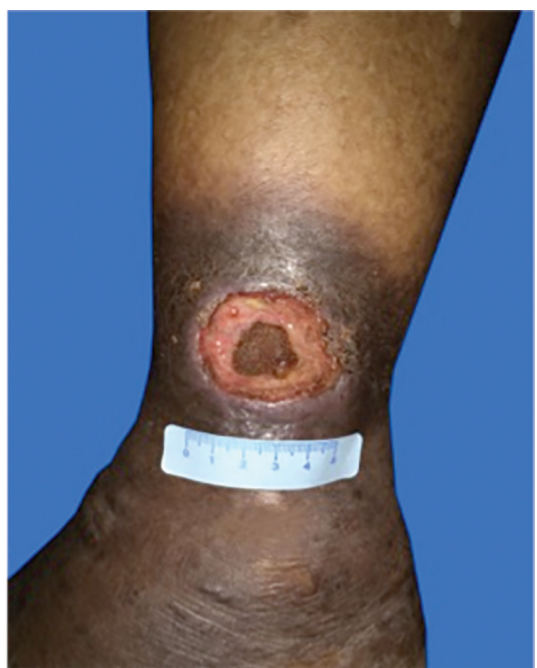

Figure 3 | Venous leg ulcer after the eighteenth LED session and wound dressing. 
wound healing for 15 minutes. This therapy was performed twice a week. Aside from that, the patient was also instructed to elevate the left leg every day. The patient was recommended to put a $0.9 \%$ saline compress on the wound for 15 minutes twice a day. Based on Indonesian Clinical Practice Guidelines, especially at Dr. Cipto Mangunkusumo Hospital, positive results on physical examination and Gram staining were considered sufficient to indicate systemic infection. Then the wound was treated with $2 \%$ fusidic acid ointment twice a day. Amoxicillin/clavulanic acid was initially administered at a dose of $3 \times 625 \mathrm{mg}$, but was then changed to tetracycline at a dose of $4 \times 500 \mathrm{mg}$ based on the result of aerobic culture and resistance. Management was provided by an internist, which included a compression bandage, rivaroxaban $1 \times 15 \mathrm{mg}$, and acetylsalicylic acid $1 \times 80 \mathrm{mg}$.

After the patient underwent LED eight times, the wound was evaluated. In the lateral part of the lower left limb region, there was an annular ulcer measuring $4 \times 4 \times 0.5 \mathrm{~cm}$, the edge of the ulcer was slightly elevated, it had a steep wall, the base was partly granulation ulcer tissue, the contents contained minimal slough, there was no induration or foul odor and there was tenderness and hyperpigmented tissue around the ulcer (Fig. 2). The VAS score was reduced from the baseline score to 2 . The results of Gram examination on the ulcer showed that there were moderate leukocytes and moderate Gram-positive cocci.

On the eighth visit, the patient received foam and hydrophobic dressings that were applied to the wound every 2 days. In the last 2 weeks, the patient started using a compression stocking. After the patient underwent LED eighteen times, the wound was evaluated. In the lateral part of the lower left limb, there was an irregularly shaped ulcer measuring $3 \times 3 \times 0.2 \mathrm{~cm}$, the edge of the ulcer was flat, it had a steep wall, the base was granulation tissue, there was no induration or foul odor and there was tenderness and hyperpigmented tissue around the ulcer (Fig. 3). The VAS score was reduced from the baseline score to 1 . The results of Gram examination on the ulcer showed that there were moderate leukocytes and a few Gram-positive cocci. There were no adverse events due to this procedure.

\section{Discussion}

From the results of the patient history, physical examination, and additional examination, the patient was diagnosed with a venous leg ulcer with secondary infection. The diagnosis was based on clinical signs in the form of a wound on the lower limb, which was painful, accompanied by occasional cramps at night. Standing for a long time, a habit related to the patient's job, could also be associated with the injury to the lower limb. The wound was accompanied by hyperpigmented surrounding tissue, static dermatitis, varicose veins, and edema in the lower left leg. The results of Gram examination of the wound showed signs of secondary infection. Doppler ultrasound examination revealed a disturbance in the veins that resulted in chronic deep vein thrombosis.

Wound evaluation was performed after the patient underwent the eighth LED treatment. It was found that the wound size decreased from $5 \times 5 \times 1 \mathrm{~cm}$ to $4 \times 4 \times 0.5 \mathrm{~cm}$. At that time, the wound still had minimal slough, and the results of Gram examination on the ulcer showed that there were moderate leukocytes and moderate Gram-positive cocci. In response to this condition, wound dressings were applied as additional treatment. A second evaluation was performed after the patient underwent the eighteenth LED session. It was found that the wound size decreased from $4 \times$ $4 \times 0.5 \mathrm{~cm}$ to $3 \times 3 \times 0.2 \mathrm{~cm}$, the base was composed of granulation tissue, and the results of Gram examination on the ulcer showed that there were moderate leukocytes and a few Gram-positive cocci. A study conducted by Caetano et al. also showed similar results regarding the effectiveness of LED in promoting wound healing (13). The necrotic tissue seen upon the first arrival of the patient had completely disappeared after the eighteenth LED session and was completely replaced with granulation tissue. The presence of secretion had disappeared. Evaluation of pain analysis using the VAS found a decrease in scores. These results indicated an improvement in the wound healing process. This finding is in line with a study conducted by Vitse et al., which showed that LED had positive effects on reducing venous ulcer pain (14).

LED is a well-accepted therapeutic tool for the treatment of infected, ischemic, and hypoxic wounds as well as other soft tissue injuries in humans and animals. Such photobiomodulation has been observed to increase mitochondrial metabolism, facilitate wound healing, and promote angiogenesis, including in the skin. LED has beneficial effects on cells by "kick-starting" them into immediately creating more adenosine triphosphate (ATP) and increasing the DNA and RNA activity. A previous study by Kim et al. showed that exposure to $660 \mathrm{~nm}$ red LED resulted in an increase in cell proliferation and migration compared to control (15), indicating its potential use as a phototherapeutic agent. The wound dressings that are applied to the wound facilitate a complete healing process in a moist and clean environment. A hydrophobic dressing can bind bacteria and remove it from wound bed. Furthermore, a foam dressing also can absorb excess exudate from the wound. Gentili et al. found that a hydrophobic dressing can reduce bacterial load and the size of a chronic lower limb wound (16).

In this case, the accelerated wound healing was also supported by other therapy approaches such as a $0.9 \%$ saline compress to moisten the wound. The administration of fucidic acid $2 \%$ ointment and tetracycline orally at the beginning also aided in eradicating secondary infection in the wound. Therefore, the wound healing process was not hampered. The patient also performed routine limb elevation each day. Moreover, oral rivaroxaban $1 \times$ $15 \mathrm{mg}$, acetylsalicylic acid $1 \times 80 \mathrm{mg}$, and a compression stocking were also beneficial for repairing venous blood circulation in the lower limb.

In this case report, we provided comprehensive treatment to achieve the best wound healing results. The wound had not completely healed at the end of this procedure, which is a limitation of this case. Written informed consent was obtained from the patient for publication of this case.

\section{Conclusions}

After the patient underwent LED eighteen times combined with wound dressings, the size, depth, and pain of the venous leg ulcer decreased. LED therapy and wound dressing have positive effects in treating venous leg ulcers and accelerating wound healing. LED and wound dressings could be chosen as a therapeutic method for wounds because this is a noninvasive treatment that is easy to arrange and use. 


\section{References}

1. Alavi A, Sibbald G, Phillips TJ, Miller OF, Margolis DJ, Marston W, et al. What's new: management of venous leg ulcer, approach to venous leg ulcers. J Am Acad Dermatol. 2016;74:627-40.

2. Rahman GA, Adigun IA, Fadeyi A. Epidemiology, etiology, and treatment of chronic leg ulcer: experience with sixty patients. Ann Afr Med. 2010;9:1-4.

3. Agale SV. Chronic leg ulcers: epidemiology, aetiopathogenesis, and management. Ulcers. 2013:1-9.

4. Xie T, Ye J, Rerkasem K, Mani R. The venous ulcer continues to be a clinical challenge: an update. Burns Trauma. 2018;6:1-7.

5. Burkhart CN, Adigun C, Burton CS. Cutaneous changes in peripheral venous and lymphatic insufficiency. In: Goldsmith LA, Katz IA, Gilchrest BA, Paller AS, Wolff $\mathrm{K}$, editors. Fitzpatrick's dermatology in general medicine. New York: McGraw Hill; 2012. p. 2110-20.

6. Fukaya E, Margolis DJ. Approach to diagnosing lower extremity ulcers. Dermatol Ther. 2013;26:181-6.

7. Dhivya S, Padma VV, Santhini E. Wound dressings-a review. Biomedicine. 2015;5:24-8.

8. Opel DR, Hagstrom E, Pace AK, Sisto K, Hirano-Ali S, Desai S, et al. Light-emitting diodes: a brief review and clinical experience. J Clin Aesthet Dermatol. 2015;8:36-44.

9. Andrade Fdo S, Clark RM, Ferreira ML. Effect of low-laser therapy on wound healing. Rev Col Bras Cir. 2014;41:129-33.
10. Tchanque-Fossuo CN, Ho D, Dahle SE, Koo E, Li CS, Isseroff RR, et al. A systematic review of low-level light therapy for treatment of diabetic foot ulcer. Wound Rep Regen. 2016;24:418-26.

11. Dungel P, Hartinger J, Chaudary S, Slezak P, Hofmann A, Hausner T, et al. Lowlevel light therapy by LED of different wavelength induces angiogenesis and improves ischemic wound healing. Lasers Surg Med. 2014;46:773-80.

12. Li S, Wang C, Wang B, Liu L, Tang L, Liu D, et al. Efficacy of low-level light therapy for treatment of diabetic foot ulcer: a systematic review and meta-analysis of randomized controlled trials. Diabetes Res Clin Pract. 2018;143:215-24.

13. Caetano KS, Frade MA, Minatel DG, Santana LA, Enwemeka CS. Phototherapy improves healing of chronic venous ulcers. Photomed Laser Surg. 2009;27:1118.

14. Vitse J, Bekara F, Byun S, Herlin C, Teot L. A double-blind, placebo-controlled randomized evaluation of the effect of low-level laser therapy on venous leg ulcers. Clin Transl Res. 2017;61:29-35.

15. Kim MS, Cho YI, Kook MS, Jung SC, Hwang YH, Kim BH. Effect of $660 \mathrm{~nm}$ lightemitting diode on the wound healing in fibroblast-like cell lines. Int J Photoenergy. 2015:1-9.

16. Gentili V, Gianesini S, Balboni PG, Menegatti E, Rotola A, Zuolo M, et al. Panbacterial real-time $P C R$ to evaluate bacterial burden in chronic wounds treated with Cutimed Sorbact. Eur J Clin Microbiol Infect Dis. 2012;31:1523-9. 